\title{
JACEK FLORCZAK
}

Université de Lódź

\section{QUELQUES REMARQUES SUR L'ISOTOPIE SÉMANTIQUE}

\begin{abstract}
Florczak Jacek, Quelques remarques sur l'isotopie sémantique [A few remarks on the semantic isotopy]. Studia Romanica Posnaniensia, Adam Mickiewicz University Press, Poznań, vol. XXX: 2003, pp. 51-72. ISBN 83-232-1270-8. ISSN 0137-2475.

The aim of this paper is to present a few theoretical remarks on the concept of semantic isotopy from the point of view of interpretative semantics and to add some examples of application of this concept in practice. In the paper we present the basic definitions of semantic isotopy (iso - identity topos - of the topic in several signification units) and describe the main classifications of this concept (systemic and discursive isotopy, intrinsic and extrinsic isotopy and others). We also discuss the fundamental semantic relations in the polyisotopic text on the levels of semes - semantic features, of sememes words which contain these features and of isotopy of a text - in which different words contain the same semantic feature. Finally we describe the basic interpretational operations that regulate the assignment of significations to words in a context and we propose a strategy of text intcrpretation oriented on isotopy.
\end{abstract}

\section{INTRODUCTION}

L'objectif de cet article sera de présenter quelques remarques théoriques sur le concept d'isotopie sémantique du point de vue de la sémantique interprétative' en l'illustrant, en même temps, de quelques exemples d'application.

Toutes les définitions d'isotopie s'organisent autour de deux genres de questions que nous allons illustrer à l'aide de cet exemple désormais classique (Greimas 1966):

$n^{\circ} 1$ Le SIchien S2aboie

$n^{\circ} 2$ Le S3commissaire S4aboie

I Il s'agit ici de la sémantique interprétative élaborée au sein des travaux de F. Rastier (1987), et liée aux travaux de Greimas, (1966), de Weinreich (1966), à la sémantique du discours (Corblin 1996) et à la linguistique du texte (Beaugrande et Dresler 1990). Elle n'est donc liéc que dans unc faible mesure à la sémantique interprétative née de la polémique avec la sémantique générative. 
Premièrement, on définit l'isotopie conformément à son étymologie («iso» «identité» et «topos» - «thème») comme récurrence d'un même trait de signification (s) dans différentes unités de signification (S) du texte. L'isotopie de la phrase $n^{\circ} 1$ s'appuie sur l'itération du trait s/animal/ dans S1 et S2. De ce point de vue, deux unités de signification différentes sont «isotopes» lorsqu'elles réalisent le même «thème», et l'isotopie est l'effet de la répétition d'un même thème dans différentes unités de signification. Deuxièmement, on entend par là un phénomène sémantique qui assure une continuité des significations et permet la désambiguïsation des mots en contexte. Dans la phrase $n^{\circ} 2$ la récurrence du trait s/humain/ établit la signification du S4 par la virtualisation du trait s/animal/ et l'actualisation du trait s/humain/. Par la mise en place d'un dénominateur commun permanent, elle garantit en plus une homogénéité au texte et rend possible sa lecture cohérente; dans la phrase $n^{\circ} 1$, nous comprenons $S 1$ et $S 2$, s o i t comme S1'mammifère domestique carnivore, canidé' e t S2'voix du chien', lorsque l'isotopie établie est /animal/, s o i t comme S1'terme d'injure désignant une personne désagréable' e t S2'invectiver', lorsque l'isotopie établie est /humain/, et il est difficile d'imaginer la lecture des significations de ces mots comme étant à la fo is Sl'mammifère canin' e t $S 2$ 'cri désagréable d'une personne' dans la phrase $n^{\circ} 1$, ou bien S3'officier de police' e $t$ S4'voix d'un chien' dans la phrase $n^{\circ} 2$.

Ces deux types de définitions donnent lieu à deux approches de la problématique de l'isotopie. Dans la première - attributive - il s'agira de définir les propriétés de lisotopie établie, à savoir la nature des unités itératives constituant cette isotopie, i.e. traits isotopants (cf. dans $\mathrm{n}^{\circ} 2$ sème générique /humain/ inhérent dans S3 et afférent dans S4) et la nature des unités dans lesquelles se trouvent ces traits isotopants, i.e. unités isotopes (cf. dans $\mathrm{n}^{\circ} 1$ deux sémèmes: Sl'chien' et S2'aboie'). Dans la deuxième - opérationnelle - il s'agira de décrire les opérations interprétatives qui (r)établissent $1(\mathrm{es})$ isotopie(s) du texte ( $\mathrm{cf}$. $\mathrm{n}^{\circ} 2$ : virtualisation de s/animal/ et actualisation de s/humain/ dans S4) et d'étudier les relations qui s'établissent entre elles (cf. $\mathrm{n}^{\circ} 1$ où les significations des $\mathrm{S} 1$ et $\mathrm{S} 2$ actualisées suivant l'isotopie /humain/ présupposent la virtualisation de leurs sens propres de l'isotopie /animal/). Nous aborderons ces problèmes dans les chapitres qui suivent.

\section{UNITÉS ET SÉQUENCES ISOTOPES}

La question est de savoir quels peuvent être (i) les types de traits isotopants contenus dans différentes unités isotopes et (ii) les types d'unités isotopes contenant ces traits isotopants. Nous commencerons par la deuxième question, et nous examinerons ces unités du point de vue de leur grandeur (mot, phrase, texte) en les analysant d'une part dans la dimension discursive et d'autre part dans la dimension systémique du langage. 
D'abord, ces unités de signification peuvent être simplement des mots. Or, le contenu d'un mot recouvre deux types de formes de contenus: son sens manifesté dans le système linguistique («lexème») et sa signification actualisée en discours («sémème»), qui désignent deux types d'unités isotopes et donnent lieu à deux types d'isotopies correspondantes: isotopie systémique, considérée comme présence d'un même trait isotopant dans différents lexèmes hors contexte, et isotopie discursive considérée comme itérativité d'un même trait isotopant dans différents sémèmes en contexte.

Dans la structure générale du système lexical ou dans son organisation hiérarchique deux plans isotopes sont à distinguer: $1^{\circ}$ le plan horizontal, où le trait isotopant est un trait générique commun aux lexèmes du même niveau et enregistré en tant qu'écart différentiel dans leur archilexème situé au niveau supérieur, p.ex.: s/pour s'asseoir/ dans S1'chaise' $\cap$ S2'fauteuil' $\cap$ S3'banc', dont l'archilexème est 'siège', $2^{\circ}$ le plan vertical, où le trait isotopant est récurrent aux lexèmes situés aux différents niveaux hiérarchiques. On peut envisager ici deux cas: (i) le plan vertical proprement dit, où le trait récurrent est (macro)générique pour tous les lexèmes de la séquence, p.ex.: s/objet/ dans $S 3$ 'chaise' $\subset \mathrm{S} 2$ 'siège' $\subset$ Sl'meuble', (ii) le plan oblique, où le trait récurrent passe du statut de «différence spécifique» au statut de "genre commun» suivant le niveau auquel se situe le lexème qui le manifeste, p.ex.: s/pour s'asseoir/ commun à S2'siège', où il est spécifique, et à $S 3$ 'chaise', où il est générique.

Le concept d'isotopie peut être élargi aussi à toute dimension hors discursive dépassant l'ordre du mot, et l'on pourra considérer comme isotopes un ensemble de phrases telles que:

$\mathrm{n}^{\circ} 3$ Mon S1destin S2est accablant

$n^{\circ} 4$ Jean pensait que son $\$ 3$ avenir S4était radieux

liées par les traits isotopants s/futur/commun aux S1 et S3, et s/jugement/commun aux prédicats S2 et S4. (Greimas, 1966). De ce fait, on pourra considérer comme isotopes les proverbes suivants qui réalisent le même thème de /relation de cause à effet/ et qui, malgré leurs sens littéraux différents, sont de ce fait commutables dans des contextes similaires:

$\mathrm{n}^{\circ} 5 \mathrm{~A}$ bon chat, bon rat

$\mathrm{n}^{\circ} 6 \mathrm{~A}$ vilain, vilain et demi

$\mathrm{n}^{\circ} 7 \mathrm{~A}$ beau jeu, beau retour

ou bien les proverbes ci-dessous qui, malgré leurs messages «latents» différents, peuvent être considérés comme isotopes, car ils contiennent les mêmes lexèmes isotopants:

$\mathrm{n}^{\circ} 8$ La nuit, tous les chat sont gris

$\mathrm{n}^{\circ} 9$ Il ne faut pas réveiller le chat qui dort

$n^{\circ} 10$ Quand le chat est parti les souris dansent 
On remarquera cependant que pour de nombreux sémanticiens, lisotopie est un concept qui relève ex definitione de la dimension discursive du langage (cf.: approche opérationnelle). De ce point de vue, on considère comme isotopes (i) d'une part, les différents sémèmes d'un texte dont les significations actualisées en contexte possèdent un même trait isotopant, (ii) d'autre part, une séquence quelconque du discours dont au moins deux sémèmes différents possèdent un ou plusieurs traits en commun. Soit l'exemple $n^{\circ} 1$ présenté formellement:

Sq: Le S1chien(s1/animal/N/s2/canin/) S2aboie(s1/animal/N/s3/voix canine/), et: $\mathrm{Sq}(\mathrm{i})=\mathrm{S} 1 / \mathrm{s} 1 / \% / \mathrm{s} 2 /^{\prime} \wedge \mathrm{S} 2 / / \mathrm{s} 1 / \% / \mathrm{s} 3 \prime^{\prime}$, ou Sq(ii) $=/ \mathrm{s} 1 / \in(\mathrm{S} 1 \wedge \mathrm{S} 2)$

En fin de compte, nous remarquerons ce qui suit. Une unité n'est pas isotope en soi et elle peut concerner différentes dimensions. D'une part, on considère comme isotopes au moins deux unités isolées contenant un trait isotopant commun, d'autre part un ensemble ou une séquence des dites unités. On peut considérer comme isotopes aussi bien une séquence de différents mots en contexte qu'un ensemble de mots en système. Dans le deuxième cas, les traits isotopants induisent des équivalences sémantiques entre les sens systémiques des lexèmes et rendent compte de relations paradigmatiques de similarité, dans le premier cas, entre les significations des sémèmes actualisées en contexte rendant compte de relations syntagmatiques de contiguiité. La sémantique interprétative ne s'intéresse qu'à la définition discursive de l'isotopie sémantique, pour laquelle elle est un des outils principaux d'interprétation du texte, laquelle isotopie relève de l'une des dimensions de l'analyse textuelle. Soit:

\section{$n^{\circ} 11$ Si jeunesse savait si vieillesse pouvait}

où l'on distingue trois dimensions d'interprétation: micro-, méso- et macrosémantique auxquelles correspondent différentes grandeurs interprétées: traits de signification - sèmes (p.ex.: s1/âge/, s/jjeune/, s3/vieux/), sémèmes - ensemble de sèmes dont se compose la signification d'un mot (p.ex.: Sl'jeunesse', qui contient /sl/ et $/ \mathrm{s} 2 /$, ou S2'vieillesse' contenant /s1/ et /s3/) et l'isotopie - itération d'un même sème dans différents sémèmes du texte (p.ex $1 / \mathrm{s} L / \in \mathrm{SI} \wedge \mathrm{S} 2$ ). Il existe une relation étroite entre toutes ces unités que l'on peut expliquer au nom du principe selon lequel les parties déterminent la signification de la totalité, de même que la totalité détermine la signification de ces parties. C'est ainsi que l'isotopie relevant du niveau macrosémantique du texte est l'effet de la récurrence d'un même sème, qui, lui, relève du niveau microsémantique, dans différents sémèmes du texte qui appartiennent, à leur tour, au niveau mésosémantique. L'isotopie du texte $n^{\circ} 11$ est basée sur l'itération du sème $\mathrm{s} 1$ dans les sémèmes $\mathrm{S} 1$ et $\mathrm{S} 2$. 


\section{TRAITS OU COMPOSANTS ISOTOPANTS}

Les traits isotopants peuvent relever de différents niveaux linguistiques:

$n^{\circ} 12$ (publicité de l'eau minérale BADOIT) Et badadi et badadoit, la meilleure eau c'est la BADOIT

$n^{\circ} 13$ (publicité d'une agence de voyage FRAM) Les vacances à la FRAMçaise et nous pouvons parler d'isotopies phonétiques, graphiques ou autres, qui désignent la sphère de signification du texte largement comprise et qui seront différemment exploitées dans des types de messages variés. Les messages radiophoniques joueront de préférence sur les isotopies phonétiques, tandis que les isotopies graphiques ne pourront être exploitées que dans les messages écrits.

Nous nous concentrons sur l'isotopie sémantique, et comme elle est l'effet de l'itération d'un même sème dans différents sémèmes du texte, il conviendra de rappeler la notion de sème et les types d'isotopies sémantiques seront établis relativement aux types de sèmes isotopants. Par sème on comprend communément l'unité de signification distinctive et pertinente réalisée à l'intérieur d'un sémème. Les types de sèmes seront dégagés selon deux critères. Le premier est celui de généricité et nous distinguons: sèmes génériques - élément du classème $(\mathrm{Cs})$, permettant de rapprocher deux sémèmes différents par un trait commun (genus proximum) qui établissent les relations de similarité et de conjonction entre les sémèmes comparés («chien» $\wedge$ «chat» $\in$ /animal/) et sèmes spécifiques - éléments du sémantème $(\mathrm{Sm})$ permettant de différencier deux sémèmes voisins par une caractéristique propre (differentia spaecifica) qui établissent les relations d'opposition ou de disjonction entre les sémèmes voisins («chien» $\rightarrow /$ canin/ $\div$ «chat» $\rightarrow$ /félin/). L'autre critère de distinction de sèmes est relatif à la distinction entre le système de la langue et d'autres types de codifications linguistiques. Si nous retenons, en fait, que le sème est un trait pertinent et distinctif d'un sémème, il peut s'avérer qu'un trait considéré comme non-distinctif pour une analyse lexicale hors contexte, peut être fort distinctif en contexte et considéré de même comme sème. Nous mettons donc dans la sphère de signification d'un sémème différents types de composants sémantiques et nous les divisons en traits inhérents et afférents, où les premiers relèvent d'une structure abstraite d'interdépendances idéalisées, basées sur les relations d'identité et d'opposition entre les mots dans le système lexical traditionnellement entendu; p.ex.: S1chien/canis/ vs S2chat /felis/, tandis que les seconds relèvent d'autres types de codifications sémantiques, et peuvent désigner certains traits associatifs relevant d'une norme sémantique supra-individuelle, i.e. traits sociolectaux, p.ex.: /fidélité/ ou /obéissance/ pour «chien», ou bien des traits occasionnels qui ne sont pas figés dans la mémoire de l'usager et dont l'actualisation n'est propre qu'aux mécanismes du discours, comme ce trait idiolectal /plat/ actualisé dans $S^{\circ}$ 'chien' dans la phrase:

$n^{\circ} 14$ En Chine, les chiens sont considérés comme un vrai délice. 
Respectivement aux dimensions discursive et systémique du langage, on parlera de sens du sémème-type $\left(\mathrm{S}^{t}\right)$ d'un lexème en système, composé de sèmes inhérents, et de signification du sémème-occurrence $\left(S^{\circ}\right)$ d'un lexème en discours, composé de sèmes (inhérents et/ou afférents) actualisés en contexte. L'isotopie discursive s'appuiera sur les sémèmes-occurrences isotopes dans lesquels quatre types de traits isotopants pourront être actualisés:

\begin{tabular}{c|c|c} 
sèmes & génériques & spécifiques \\
\hline inhérents & $/ \mathrm{s} 1 /$ & $/ \mathrm{s} 3 /$ \\
\hline afférents & $/ \mathrm{s} 2 /$ & $/ \mathrm{s} 4 /$
\end{tabular}

et en fonction desquels différents types d'isotopies pourront être (r)établies. Soit dans le titre du roman de Stendhal:

\section{$n^{\circ} 15$ Le S1rouge et le S2noir}

le /s1/ commun aux S1 et S2 est/couleur/ et il établit l'isotopie générique inhérente. Les S1 vs S2 sont opposés par s3/couleur sang/ pour S1 et s3/couleur charbon/pour $S 2$. Des afférences contextuelles et la connaissance des normes sociales permettent de construire des sèmes afférents. Le /s2/ commun aux S1 et $\mathrm{S} 2$ sera dénommé /couche sociale/ ou /carrière/ et il établit l'isotopie générique afférente. Les S1 vs S2 sont opposés par les sèmes spécifiques afférents: s4/armée/ ou /militaire/ pour S1 et s4/clergé/ ou /ecclésiastique/ pour S2.

En somme, ces quatre types de sèmes isotopants donnent lieu aux types d'isotopies suivants. Conformément à la distinction entre sèmes génériques et spécifiques, on distinguera: isotopies génériques (/animal/ dans la phrase $\mathrm{n}^{\circ} 1$ ), spécifiques (/canin/ dans la même phrase) et mixtes (/nez/ dans le nez aquilin). Conformément à la distinction entre sèmes inhérents et afférents on distinguera isotopies inhérentes (/animal/ dans la phrase $n^{\circ} 1$ ), afférentes (/humain/ dans la même phrase), et mixtes (/humain/ dans la phrase $n^{\circ} 2$ ).

\section{OPÉRATIONS ET RELATIONS ISOTOPIQUES}

Toute lecture isotopique d'un texte quelconque est précédée d'opérations interprétatives élémentaires qui rendent compte du processus de variations sémiques en contexte et des règles d'assignation de la signification aux sémèmesoccurrences. La signification du mot en discours est résultante de ces traits codifiés dans la norme sémantique (ensemble de tous les traits sémantiques supraindividuels) et du contexte de son emploi (ou son interprétant largement compris, i.e. contexte linguistique et extralinguistique ainsi que locuteur), dans lequel il actualise (Ac) et/ou virtualise $(\mathrm{Vr})$ les traits inhérents $\left(s_{i}\right)$ et/ou afférents $\left(s_{a}\right)$. Soit:

$\mathrm{n}^{\circ} 16 \mathrm{La}$ parole est d'argent, mais le silence est d'or 
où, par cohérence interprétative, dans $S_{1}^{\circ}$ 'argent' et $S_{2}^{0}$ 'or' sont virtualisés leurs traits inhérents communs /concret/, /métal/ et différenciateurs /blanc/ pour $S_{1}^{0}$ et /jaune/ pour $S_{2}^{\circ}$, et sont actualisés les traits tels que /abstrait/, /évaluation/ ou /préciosité/ communs aux $S_{1}^{o}$ et $S_{2}^{0}$ et leurs traits différenciateurs $S_{1}^{o} /$ moins précieux/vs $S_{2}^{0}$ /plus précieux/. Les mêmes opérations gèrent l'interprétation de la signification du $S^{O^{\prime}}$ chien' dans l'exemple $n^{\circ} 14$, où dans le contexte de $S^{O^{\prime}}$ délice' son trait inhérent /animé/ est virtualisé et on actualise le trait afférent/plat/. Il apparaît donc ici un nouveau principe de pertinence sémantique en contexte. Ce qui est pertinent relativement au système fonctionnel de la langue peut être virtualisé en contexte, ce qui est actualisé en contexte en tant que trait pertinent peut être non pertinent relativement au système fonctionnel de la langue. Dans la phrase:

$n^{\circ} 17$ Guillaume était la femme dans le ménage

on virtualise le trait inhérent de /sexe féminin/ dans $S^{O}$ 'femme' et l'on actualise les traits afférents relatifs au /caractère ou comportement particulier propre aux femmes/. Dans cette phrase le $S^{\circ}$ 'femme' ne change pas de sens (systémique) mais assume une (autre) signification contextuelle. Autrement dit, son trait inhérent /sexe féminin/ n'est pas nié, mais justement... virtualisé. Et c'est ainsi que l'on conçoit le concept de virtualisation. Les traits afférents ainsi actualisés peuvent avoir le caractère continu. Il n'en reste pas moins vrai que c'est justement ce type /„particulien» ou «caractéristique» de comportement propre aux femmes/ qui sera considéré ici comme étant le trait nécessaire et suffisant pour la description de la signification actualisée dans ce sémème-occurrence. Pour la sémantique interprétative, le principe de pertinence sémantique rejoint donc celui d'économie sémiotique qui s'exprime par la tendance à optimiser le coût sémiotique de l'échange langagier dans le bilan des pertes (en termes de précision d'expression du contenu) et des profits (en termes de concision d'expression du continu) auxquels consentent les acteurs de cet échange relativement aux besoins de la situation de communication. Ce principe de pertinence est aussi lié à celui d'économie descriptive qui consiste à éliminer de la description des traits actualisés, les traits qui n'ont pas de pertinence contextuelle et à restreindre leur description aux traits suffisants et nécessaires, lesquels sont de ce fait nécessairement continus et donc non analysables en termes d'unités discrètes.

Fondamentalement, les opérations qui permettent l'actualisation et la virtualisation des sèmes sont l'assimilation (As) et la dissimilation (Ds) des sémèmes. La première consistera dans l'actualisation d'au moins un trait sémantique identique aux deux sémèmes voisins en apparence contradictoires ou incompatibles, et elle induit la relation de conjonction $(\wedge)$ entre les significations de différents sémèmesoccurrences à partir de la relation d'identité entre les traits actualisés dans ces sémèmes. La deuxième consistera dans l'actualisation d'une différence entre deux sémèmes voisins sémantiquement proches l'un de l'autre, voire identiques, et elle 
induit la relation de disjonction $(\div)$ entre ces mots par l'actualisation de leurs traits réciproquement distinctifs ou opposés. On peut l'illustrer à l'aide des syntagmes tautologique et contradictoire ci-dessous 2 :

$$
\begin{aligned}
& \mathrm{n}^{\circ} 18 \text { Printemps(S1) printanier(S2) As }\left\{\mathrm{S} 1 \wedge \mathrm{S} 21^{/ \mathrm{s} 1 /} ; \mathrm{Ds}\left\{\mathrm{S} 1^{/ \mathrm{s} 2 /}+\mathrm{S} 2^{/ 33}\right\}\right. \\
& \text { L'hiver(S2) en été(S1) }
\end{aligned}
$$

où: s1/saison de l'année/, s2/période (choisie)/, s3/temps météo (avec propriétés caractéristiques)/

Grâce aux opérations interprétatives ci-dessus nous pouvons (r)établir différentes isotopies d'un texte et décrire leurs relations sém(ém)iques réciproques. Soit cet exemple de la phrase $n^{\circ} 19$ : Les gens se divisent en deux catégories: les politiciens et les gens honnêtes, présentée de manière graphique:

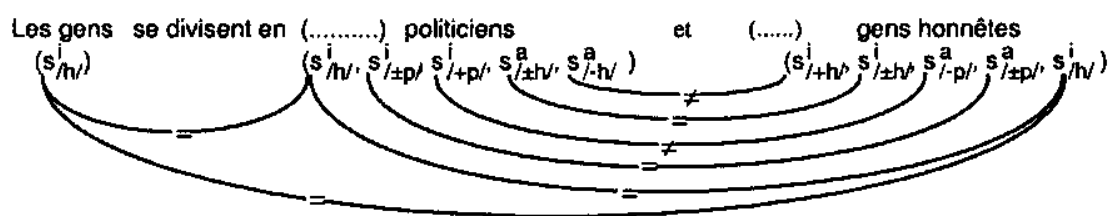

où: $\mathrm{h}=$ humain, $\pm \mathrm{h}= \pm$ honnête, $+\mathrm{h}=$ honnête, $-\mathrm{h}=$ non honnête,

$\pm p= \pm$ politicien, $+\mathrm{p}=$ politicien, $-\mathrm{p}=$ non politicien

Grossièrement, les relations d'identité $(\Rightarrow)$ entre les occurrences du sème isotopant induisent les relations d'équivalence entre les sémèmes-occurrences qui les incluent, et établissent les isotopies du texte par assimilation du sème isotopant, tandis que les relations de différence $(\neq)$ entre les occurrences des sèmes opposés établissent les allotopies du discours par dissimilation des sémèmes. Si donc en système, les sémèmes ne manifestent que leurs traits inhérents, le contexte peut virtualiser des sèmes inhérents pour actualiser des sèmes afférents, et comme les isotopies discursives sont des phénomènes contextuels, elles comprennent indiffé-

${ }^{2}$ On voit bien que le postulat de la primauté de la norme sur le discours énoncé au début du chapitre ne signifie pas que cette norme détermine l'acceptabilité des emplois des mots en discours. Car si la connaissance des traits normatifs du mol détermine les règles de ses cmplois acceptables, certains de ses emplois peuvent transgresser ces règles (volontairement ou non) en vue d'énoncer (ou de suggérer) des significations discursives occasionnelles. C'est ainsi que dans (i) Garçon bien roulé on "donne à comprendre" que le garçon en question a /des traits de féminitél, étant donnć que S'bien roulé' possède une restriction sélective /pour femme/ attribuée à ce sémème dans sa concaténation avec S'personne', dans (ii) Fermer la lumière on virtualise le trait /espace/ inhérent à S'fermer', dans (iii) Guerre humanitaire on virtualise le trait /non humanitairc/ afférent à S'guerre', qui est en opposition avec S'humanitaire'. Et ces cmplois des mots non conformes à la norme sémantique peuvent résulter de la loi du moindre effort (certains diront de la négligence) du locuteur (cf. ii), de la sphère d'emploi suggestif (cf. i), ou bien des tentatives de manipulation linguistique: (cf. iii) la guerre n'est pas humanitaire mais la guerre humanitaire n'est plus une guerre. 
remment des traits inhérents eVou afférents. Dans $n^{\circ} 14 S_{1}^{\circ}$ 'chien' et $S_{2}^{\circ}$ 'délice' contiennent le sème isotopant /cuisine/ ou /culinaire/ qui a le statut de trait afférent sociolectal dans $S_{2}^{o}$ et celui du trait afférent occasionnel (idiolectal) dans $S_{1}^{o}$. Dans $n^{\circ} 20$ :

$\pi^{\circ} 20$ Les Dupont mènent la grande vie: caviar et champagne tous les jours, alors que nous on doit se contenter de pain et d'eau.

sont actualisées deux isotopies génériques par la récurrence des traits isotopants inhérent $S_{\text {in }}^{g}$ alimentation/ et afférent $S_{a f}^{g}$ /produit tluxueux/ dans les sémèmes isotopes $S_{1}^{\circ}$ 'caviar', $S_{2}^{0}$ 'champagne', $S_{3}^{0}$ 'pain' et $S_{4}^{\circ}$ 'eau', et deux isotopies spécifiques par la récurrence d'un sème afférent sociolectal $S_{\mathrm{af}}^{\mathrm{s}} /+$ luxueux/ commun aux $S_{1}^{o}$ et $S_{2}^{o}$, et par celle d'un sème afférent contextuel $S_{\text {af }}^{s} /$-luxueux/ commun aux $S_{3}^{o}$ et $S_{4}^{o}$, qui sont à leur tour en relation de disjonction. Les traits inhérents permettant de décrire les particularités alimentaires de chacun de ces produits passent au second plan dans la lecture de ce message.

Un texte peut donc contenir plusieurs isotopies à la fois, et on parle le plus souvent de textes bi- ou polyisotopes, en attribuant cette notion aux textes qui contiennent deux ou plusieurs isotopies génériques. Différentes relations peuvent s'établir entre ces isotopies et différents parcours de lectures peuvent être envisagés. C'est ainsi que la séquence $\mathrm{n}^{\circ} 1$ Le chien aboie sera dite monoisotope lorsqu'elle sera énoncée à propos d'un chien, suite à la récurrence du sème générique /animal/. Elle peut être énoncée également à propos d'un homme ou bien elle peut être considérée comme ambiguë dans la situation où son contexte (interprétant) ne sera pas suffisamment précis et il maintiendra alors l'absence de choix entre l'une des significations des sémèmes-occurrences; une telle séquence sera dite bi-isotope: /animal/ et/ou /humain/. Dans ce cas, nous avons affaire aux isotopies homo-positionnelles, car les mêmes sémèmes du texte sont indexés à la fois sur deux isotopies différentes et nous pouvons distinguer les deux cas suivants. Premièrement, l'isotopie /humain/ peut être actualisée, tandis que lisotopie /animal/ est virtualisée. En ce cas, les isotopies sont en relation de disjonction exclusive, mais les significations actualisées présupposent les significations virtualisées, ce qui veut dire que l'interprétation du texte ne s'appuie pas uniquement sur l'isotopie actualisée, mais aussi sur elle. Nous l'illustrerons à l'aide des textes publicitaires suivants:

$n^{\circ} 21$ Pub d'une agence d'assurances: - Mój tata jest agentem - Może takim jak James Bond..? - Nie, jest agentem «Compensy Życie».

$n^{\circ} 22$ Pub des magasins de bricolage «Praktiker»: Przyszedłem, bo koledzy mi powiedzieli, że mogę tu dostać fajne płyty [...] i kupię sobie jeszcze ten kompakcik.

Dans les textes ci-dessus deux lectures possibles des sémèmes mis en relief sont envisageables, dont la première est virtualisée. Dans $n^{\circ} 21$, le processus de 
réinterprétation de la signification est indiqué par le contexte linguistique; dans la première occurrence de «agent» on actualise la signification $S^{\circ}$ 'agent secret', dans la seconde celle-ci est virtualisée et c'est la signification 'agent d'assurances' qui est actualisée. Dans le $n^{\circ} 22$, le processus de réinterprétation de la signification est indiqué par le contexte extralinguistique: le texte est énoncé par un rocker dans un plan américain, et les significations actualisées sont celles de 'disques vinyle' pour S1'płyty' et de 'compact disque' pour S2'kompakcik', et ce n'est qu'après qu'apparaît un plan général des rayons du magasin, où l'on comprend que les significations actualisées dans $\mathrm{S} 1$ et de $\mathrm{S} 2$ sont celles de 'panneaux de bois' et de 'cuvette de w.c.'.

Dans l'autre cas, dans un contexte ou interprétant ambigu, les deux isotopies à la fois peuvent être considérées comme étant actualisées, et aucune d'elles ne sera virtualisée, comme dans ces slogans publicitaires:

\section{$n^{\circ} 23$ Slogan publicitaire des hypermarchés "Mammouth»: Mammouth écrase les prix. \\ $n^{\circ} 24$ Slogan publicitaire pour la barre chocolatée «Mars»: Un coup de barre, et ça repart}

En ce cas, les deux isotopies des textes analysés ( $\mathrm{n}^{\circ}$ 23: /animal/ et /magasin/, $\mathrm{n}^{\circ}$ 24: /non matériel/ et /matériel/) sont en relation de disjonction inclusive, ce qui permet de relier les deux significations des mots ambigus par la relation de conjonction ( $\mathrm{n}^{\circ} 23$ : «Mammouth» comme 'animal pachyderme' et 'chaîne d'hypermarchés', «écra-ser» comme 'broyer' et 'anéantir', $\mathrm{n}^{\circ} 24$ : «coup de barre» comme 'baisse d'énergie' et 'barre chocolatée').

Les relations entre les isotopies établies sont les plus diverses, et elles peuvent être analysées à tous les niveaux du texte, soit en termes de relations micro-, entre les sèmes (isotopants), méso-, entre les sémèmes (isotopes) et macrosémantiques, entre les isotopies. Pour ce faire, nous nous servirons de deux critères de distinction d'isotopics: critère de lexicalisation et critère positionnel. Le premier concerne la présence de sémèmes isotopes dont les traits isotopants sont inhérents à ces sémèmes. Une isotopie est donc lexicalisée lorsque les sémèmes qu'elle indexe incluent son sème isotopant en tant que trait inhérent (p.ex.: isotopies /animal/ dans $n^{\circ} 1$ ou /alimentation/ dans $n^{\circ} 20$ ), sinon elle n'est pas lexicalisée (p.ex.: /humain/ dans $n^{\circ} 1$ ou /caractère \pm luxueux/ dans $n^{\circ} 20$ ). Le critère positionnel concerne la position syntagmatique des sémèmes indexés sur les isotopies différentes. En cas d'isotopies homopositionnelles, les mêmes sémèmes du texte sont indexés à la fois sur deux isotopies différentes (p.ex. isotopies /humain/ et /animal/ dans $\mathrm{n}^{\circ} 1$ ou /alimentation/ et /caractère +luxueux/ dans $n^{\circ} 20$ ), en cas d'isotopies hétéropositionnelles, les isotopies différentes indexent les sémèmes différents (p.ex. /âge/ et $/$ modalité/ dans $n^{\circ} 11$ ou /cécité/ et /règne/ dans $n^{\circ} 25$ ). Ces quatre types d'isotopies sont strictement corrélés et rendent compte des relations isotopiques fondamentales d'un texte quelconque (cf. approche attributive). A partir de ces deux critères, nous 
présenterons ci-dessous deux cas remarquables, auxquels correspondent deux types de parcours interprétatifs différant suivant le type de relation isotopique manifesté et qui indiqueront des éléments d'opérations interprétatives relevant de la lecture isotopique d'un texte (cf. approche opérationnelle).

4.1. Le premier cas analysé $e^{3}$ est celui où un texte contient deux isotopies génériques homopositionnelles dont l'une est lexicalisée (Isotopie 1) et l'autre ne l'est pas (Isotopie 2).

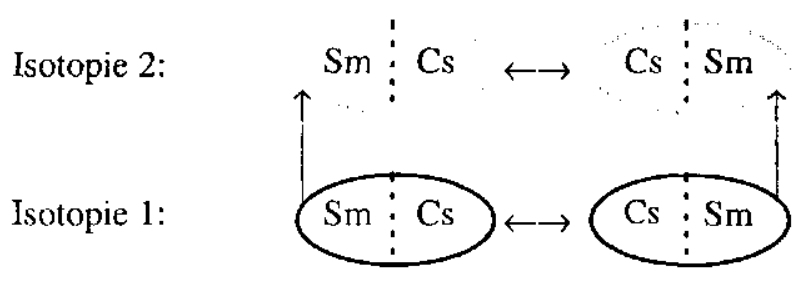

Soit toujours l'exemple $n^{\circ} 1$ Le chien aboie où nous sommes en présence de deux isotopies génériques: l'isotopie 2 constituée, en présence d'un interprétant extratextuel, par la récurrence du trait/humain/ et l'isotopie1/animal/, en opposition avec la précédente. Ces deux isotopies ont un statut différent. L'isotopie 1 est constituée par la récurrence d'un trait inhérent; l'isotopie 2, par celle d'un trait afférent. Les sémèmes indexés sur l'isotopiel sont lexicalisés; les sémèmes indexés sur l'isotopie 2 ne le sont pas: pour les lexicaliser, il faut procéder à une réécriture $(\rightarrow)$ interprétative des sémèmes isotopes de l'isotopie lexicalisée sur l'isotopie non lexicalisée de manière à ce que les sémèmes réécrits incluent le sème isotopant de l'isotopie non lexicalisée en tant que trait inhérent; ainsi Greimas (1966) réécrit, de fait, 'chien' par |'secrétaire'|. On a alors la structure sémantique suivante:

Isotopie 1 /animal/:

Sémèmes anisotopes:

Isotopie 2/humain/:

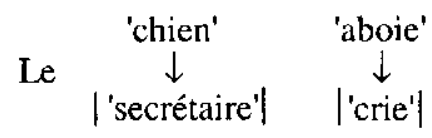

Le sémème réécrit lexicalise (LS) le sème isotopant $(\mathrm{Cs})$ de l'isotopie non lexicalisée et des éléments du sémantème $(\mathrm{Sm})$ communs aux sémèmes lexicalisé ct réécrit. Dans notre cas, le sémème 'aboie' de l'isotopie lexicalisée contient les sèmes /voix/, /forte/, /chien/, /animal/. Sa réécriture (RS) sur l'isotopie /humain/ fait virtualiser le sème /chien/ et nous obtenons une molécule sémique composée de sèmes: /voix/, /forte/, /humain/. La lexicalisation de cette molécule est la dernière

${ }^{3}$ Dans les graphiques ci-dessous les ovales pleins figurent les sćmèmes lexicalisés et les ovales en pointillés ceux qui ne le sont; $\mathrm{Cs}=$ classc̀me, $\mathrm{Sm}=$ sćmantème; graphiques empruntés à $\mathrm{F}$. Rastier (1987). 
étape de l'opération de réécriture (aboie $\rightarrow \mid$ 'cri' $\mid \div \mathrm{Sm} /$ voix/ $\wedge \mathrm{Sm} /$ forte/ $\wedge$ Cs/humaine/ $)^{4}$. Soit, graphiquement:

Isotopie 1 /animal:

'aboie' (/voix/, /forte/, /chien/)

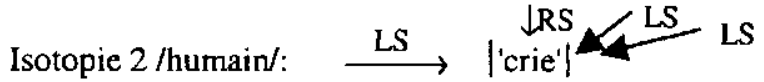

Dans ce cas, les sémèmes de l'isotopie 2 ne peuvent être lexicalisés que dans un autre texte que celui qui contient les sémèmes de l'isotopie 1. Cet autre texte a alors le statut d'un texte-but ou d'une interprétation, et nous en donnons d'autres exemples analogues:

$n^{\circ} 15$ Isotopie $1 /$ couleur/:

Isotopie 2 /couche sociale/:
Le

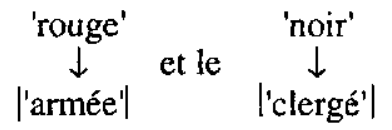

$n^{\circ} 25$ Isotopie $1 /$ cécité/: Isotopie 2 /caractère/:

Il convient d'avertir contre une lecture qui n'accepterait que la réalisation de l'isotopie 2, i.e. isotopie actualisée. Forcer à ce choix exclusif, c'est poser un faux problème, car, si l'on avait «choisi» de ne lire que la seconde isotopie, à l'exclusion de la première, il serait impossible dinférer tous les traits sémantiques de la première isotopie sur la seconde, ce qui constitue justement la caractéristique des discours plurivoques et des lectures poly-isotopes. Dans l'exemple $n^{\circ} 1$, on infère sur la seconde isotopie le /caractère péjoratif/ associé au «chien», ainsi que d'autres de ses /traits particuliers/. Si donc, en présence d'un interprétant approprié, on peut lire l'isotopie 2 /humain/, la lecture de l'isotopie 2 présuppose la lecture de l'isotopie 1, et $S^{O^{\prime}}$ chien' "connote» la signification actualisée justement parce qu'il «dénote» la signification virtualisée. On dira donc de manière générale que l'interprétation de cet énoncé ne se restreint pas à sa réécriture $\rightarrow$ l'cette personne

${ }^{4}$ Formellement, les réécritures peuvent être de nature diverse. La plus simple concerne deux sémèmes: un lexicalisé et un réécrit: 'S' $\rightarrow \mid$ 'S' ('aboie' $\rightarrow \mid$ 'crie'|). Dans les réécriturcs plus complexes plusieurs sémèmes-source peuvent être réćcrits par un sćmème-but : ' $S_{1}, S_{2}, \ldots S_{n}$ ' $\rightarrow \mid$ 'S'|: on parlera dans ce cas de condensation ('automne de la vie' $\rightarrow$ |'vieillesse'|). Lorsque le nombre de sémèmes-but est supérieur à celui de sémèmes-source: ' $S$ ' $\rightarrow\left|S^{\prime}, S^{\prime \prime}, \ldots S^{n}\right|$, on parlera d'expansion ('caviar' $\rightarrow$ |'produit luxueux'|). Les réćcritures peuvent concerner, enfin, des cas où des ensemble de sèmes sont réécrits par d'autres ensembles de sèmes: ' $S_{1}, S_{2}, \ldots S_{n}{ }^{\prime} \rightarrow\left|' S^{\prime}, S^{\prime \prime}, \ldots S^{n^{\prime}}\right|$ ('Au royaume des aveugles, les borgnes sont rois' $\rightarrow$ |'Les médiocres brillent lorsqu'ils se trouvent parmi les sots'|, cf. Petit Robert). Ces réćcritures peuvent porter sur des séquences linguistiques complexes et même sur des textes entiers. Ce dernier type de réécriture correspond à l'intergrétation traditionnelle. 
désagréable pousse des cris déplaisants', mais qu'elle consiste aussi dans la description des relations entre ces isotopies à tous les niveaux d'analyse textuelle.

4.2. Le deuxième cas analysé est celui où un texte contient deux isotopies génériques hétéropositionnelles et les deux sont lexicalisées, ce qui peut être présenté graphiquement:

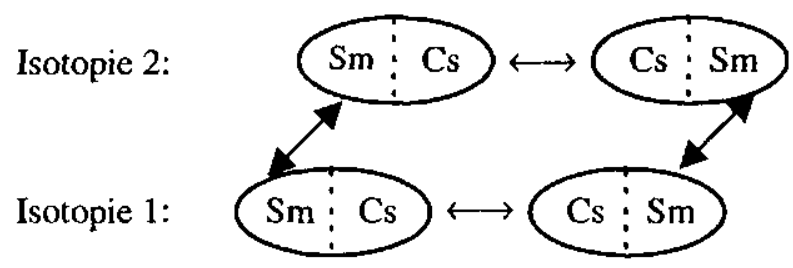

Il existe une différence de nature qualitative évidente entre le cas précédent et celuilà. Dans les exemples précédents, une isotopie était constituée par la récurrence d'un sème inhérent, une autre par celle d'un sème afférent, et les lexèmes de la première étaient lexicalisés et ceux de la seconde ne l'étaient pas. Dans ce cas-ci, les deux isotopies sont constituées par les sèmes isotopants inhérents aux sémèmes du texte et les sémèmes des deux isotopies sont lexicalisés. Nous illustrerons ce cas à l'aide de l'exemple $n^{\circ} 26$ :

$n^{\circ} 26$ L'évêque est aux fidèles comme le berger au troupeau

Le syntagme contient deux isotopies inhérentes: /religion/ et /agriculture/ que l'on peut appeler également: /église/ et /campagne/, les deux isotopies étant entièrement lexicalisées:

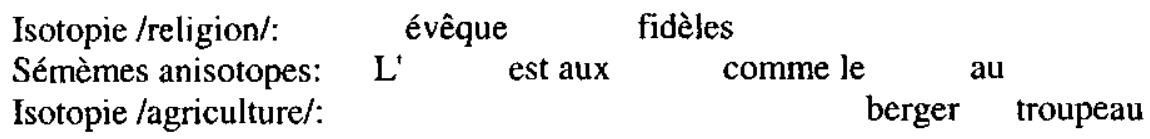

Dans ce cas, les opérations interprétatives ne comportent pas de réécritures, mais seulement des extractions de sèmes communs. Autrement dit, aucun sémème n'est réécrit par aucun autre, mais tous les sémèmes du syntagme sont associés les uns aux autres par la relation de compatibilité entre leurs sèmes spécifiques. Le parcours interprétatif qui permet d'actualiser leurs sèmes comporte deux étapes principales: lidentification du ou des sèmes génériques qui les indexent sur des isotopies différentes et l'identification des sèmes spécifiques communs. Soit, schématiquement:

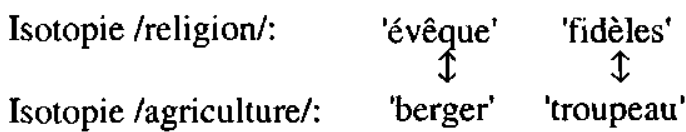


Le parcours interprétatif consistera donc dans l'association $(\leftrightarrow)$ de sémèmes lexicalisés chacun sur une isotopie différente, afin d'identifier les éléments communs de leurs sémantèmes, p.ex. /guide/ ou /supériorité/ pour: $S^{0^{\prime}}$ évêque' $\leftrightarrow$ $S^{o^{\prime}}$ berger' ou bien /docilité/ pour: $S^{0^{\prime}}$ troupeau' $\leftrightarrow S^{0^{\prime}}$ fidèles ${ }^{\prime 5}$. Et les extractions des sèmes communs des sémèmes associés formulent des éléments d'une interprétation.

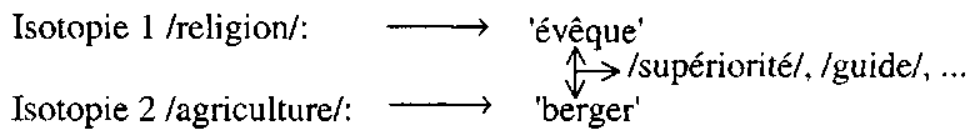

4.3. Tous les types de relations isotopiques et de parcours interprétatifs correspondants s'appuient sur quelques types d'équivalences communes à tous les types de relations isotopiques analysées. Si donc on observe des différences notables entre la nature des sèmes isotopants inhérents et/ou afférents ou entre la nature des sémèmes isotopes lexicalisés et/ou réécrits, dans tous ces cas, les isotopies du texte s'appuient sur les relations d'identité entre les occurrences du sème isotopant et induisent des relations d'équivalence entre les sémèmes qui les incluent. Les sémèmes isotopes inclus dans ces isotopies peuvent être connectés les uns aux autres de telle sorte qu'il y ait une relation d'incompatibilité entre au moins un de leurs traits génériques, et une relation d'identité entre au moins un de leurs traits spécifiques, quil s'agisse de la connexion par association où par réécriture. Tous les types d'isotopies susmentionnées peuvent donc se constituer en réseaux d'isotopies rendant compte des relations sémiques entre les sémèmes qu'elles indexent, et nous distinguerons deux types d'équivalences: horizontales qui concernent les sémèmes réitérant un même trait isotopant générique (p.ex.: $\mathrm{n}^{\circ} 1$ : 'chien' $\stackrel{\text { lanimal/ }}{\longleftrightarrow}$ 'aboie' et |'secrétaire' $\stackrel{\text { hhumain' }}{\longleftrightarrow}$ |'cri'| en cas d'isotopies homopositionnelles, ou $n^{\circ} 26:$ 'évêque' $\stackrel{\text { /religion/ }}{\longleftrightarrow}$ 'fidèles' et 'berger' $\stackrel{\text { lagriculture/ }}{\longleftrightarrow}$ 'troupeau' en cas d'isotopies hétéropositionnelles), et verticales qui concernent les sémèmes indexés sur deux (ou plusieurs) isotopies génériques différentes mais comportant des sèmes spécifiques communs (p.ex.: $n^{\circ} 1:$ 'aboie' $\stackrel{\text { /voixl }}{\longrightarrow}$ |'cri'| en cas d'isotopie non lexicalisée ou $n^{\circ} 26$ : 'évêque' $\stackrel{\text { iguidel }}{\longleftrightarrow}$ 'berger' en cas d'isotopie lexicalisée). Un texte qui comporte plus d'une isotopie générique peut donc comporter plusieurs isotopies spécifiques qui traduiront les relations de connexion entre les sémèmes du texte. Et si l'on s'en tient aux relations d'identité entre sèmes, ou d'équivalence entre sémèmes, on peut obtenir cette représentation schématique:

${ }^{5}$ De manière analogue aux connexions par réécriture, les connexions par association peuvent s'effectuer entre deux sémèmes: ' $S 1$ ' $\leftrightarrow$ 'S2', entre un et plus d'un sémème: 'S1' $\leftrightarrow S 2$ ', $S 2$ ", $\ldots S 2^{n}$ ', ou entre plusieurs sémèmes: ' $S 1_{1}, S 1_{2}, \ldots S 1_{n}$ ' $\leftrightarrow$ 'S2', $S 2$ ", ...S2 ${ }^{n}$ ' (cf. métaphore filće). 


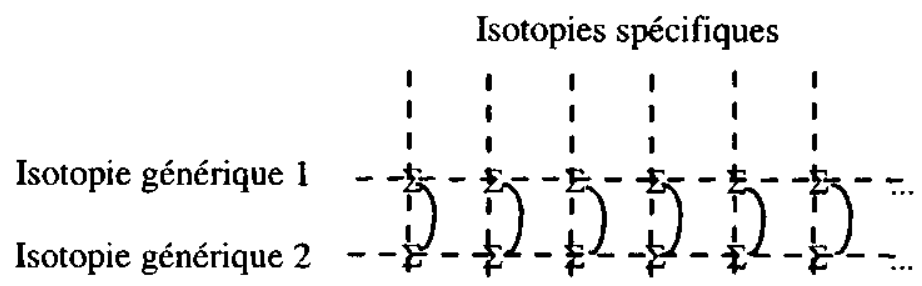

A ces deux types d'équivalences correspondent deux types de parcours interprétatifs qui concernent, d'une part, la récurrence des traits isotopants communs aux sémèmes différents d'une même isotopie générique, et, d'autre part, celle des traits isotopants spécifiques communs aux sémèmes connectés et indexés sur les isotopies génériques différentes. Soit:

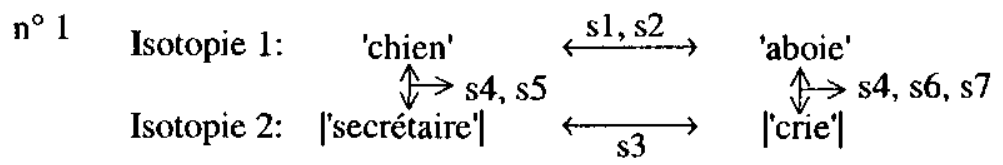

où: s1/animal/, s2/canin/, s3/humain/, s4/péjoratif/, s5/servilité/, s6/voix/, s7/fort/.

$\mathrm{n}^{\circ} 26$ Isotopie 1: 'évêque' $\stackrel{s 1, \mathrm{~s} 2}{\longleftrightarrow}$ 'fidèles'

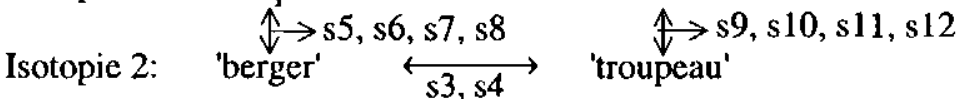

où: s1/religion/, s2/église/, s3/agriculture/, s4/campagne/, s5/humain/, s6/singulier/, s7/supérieur/, s8/guide/, s9/animé/, s10/pluriel/, s 11/subordonné/, s12/docile/.

Les isotopies qui établissent les équivalences horizontales pourront se constituer, à leur tour, en réseau d'isotopies subordonnées à une isotopie de généricité supérieure, i.e. isotopie hypéronymique, subsumant de même tous les sémèmes isotopes des isotopies différentes, p. ex., /animal/ $\wedge$ /humain/ $\rightarrow$ /animé/ dans $\mathbf{n}^{\circ} 1$, /église/ $\wedge$ /campagne/ $\rightarrow$ /culture/ ou /mode de vie/ dans $n^{\circ} 26$. En somme, le principe du réseau s'appuie sur le simple fait qu'un seul et même sémème peut être indexé sur plusieurs isotopies différentes et qu'une seule et même isotopie peut connecter plusieurs sémèmes différents. Pour la théorie de l'isotopie, le texte paraît donc comme un réseau d'isotopies enchevêtrées les unes aux autres, et elle n'a pas pour objectif de privilégier l'une des isotopies établies au détriment des autres, jugées non essentielles, voire exclues de la description. Elle ne réduit pas la signification du texte à une isotopie; elle cherche à décrire l'interrelation des isotopies différentes, car la signification du texte, et les interprétations qu'on peut en proposer, dépendent pour une part importante du réseau de ces interrelations. On pourra admettre que la description de ces réseaux constituera la dernière étape de la stratégie interprétative orientée sur l'isotopie. 


\section{INTERPRÉTATIONS ET LECTURES D'TSOTOPIES}

Les analyses «isotopiques» des textes ci-dessous rendent compte avant tout de différentes relations sémantiques établies dans un texte poly-isotope. Pour renverser cet ordre «descriptif» dominant jusqu'ici, nous proposerons à partir des remarques précédentes un modèle de stratégie interprétative rendant compte d'une lecture isotopique d'un texte. Généralement, on peut distinguer deux étapes lors d'une lecture isotopique. La première consiste en une décortication des sèmes inhérents aux sémèmes du texte et suppose leur décomposition préliminaire en traits microsémantiques. Elle correspond à une démarche inductive qui procède des sémèmes du texte à l'établissement de son (ses) isotopie(s) par une recherche des traits sémantiques communs aux sémèmes-occurrences. Cette démarche est propre à la lecture passive des isotopies du texte où le point de départ réside dans une redondance de certains sémèmes, et qui consiste à lister les sémèmes isotopes indexés sur telle ou telle isotopie par la récurrence de leur traits génériques isotopants, ce qui peut être présenté à l'aide du graphique suivant:

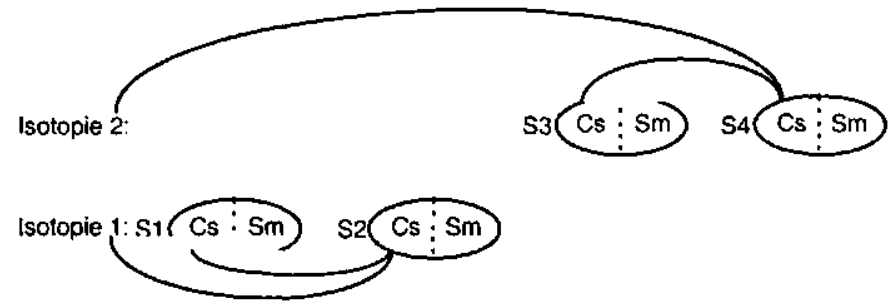

La deuxième étape va dans le sens inverse: des isotopies du texte à ses sémèmes. C'est donc une démarche déductive. Elle consiste à reprojeter l'(les) isotopie(s) établie(s) sur des sémèmes qui nc la lexicalisent pas directement. En cela elle est donc une lecture active qui consiste à comparer les isotopies génériques préalablement établies et à analyser les réseaux isotopiques qu'elles pourront former. Elle consiste donc à réécrire, là où c'est possible, des sémèmes lexicalisés d'une isotopie, sur une autre non lexicalisée, et/ou à associer des sémèmes lexicalisés sur des isotopies différentes en vue de sélectionner des traits sémantiques communs aux sémèmes connectés. Ceci peut être présenté à l'aide du graphique suivant:

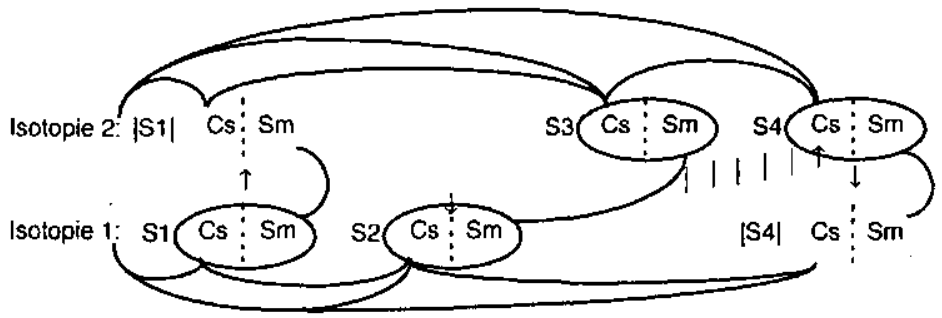


Il existe des situations où le passage à la lecture active est obligatoire, notamment au niveau pragmatique, si l'on veut comprendre le contenu du message (cf. $\mathrm{n}^{\circ} 1$ le chien aboie). Dans d'autres discours plurivoques (poly-isotopes), notamment sur le plan poétique, nous pouvons «nous permettre» de ne pas procéder à cette lecture active. Nous nous trouvons, cependant, dans la situation d'une suspension sémantique et nous ne dévoilons pas pleinement la signification du message.

Les exemples de ces parcours interprétatifs ont déjà été amplement illustrés tout au long de notre travail (notamment dans $\$ 4.1-\$ 4.3$ ). Pour les récapituler nous analyserons le texte:

$n^{\circ} 27$ Bergère, ô tour Eiffel, le troupeau des ponts bêle ce matin

qui contient deux isotopies génériques: homo- et hétéropositionnelles lexicalisées partiellement. On envisage ici une isotopie constituée par la récurrence des traits isotopants /ville/ et/ou /inanimé/ dans les sémèmes 'tour Eiffel' et 'ponts' et une autre constituée par celle des traits /campagne/ et/ou /animé/ dans: 'bergère', 'troupeau', 'bêle'. La première étape de cette lecture isotopique consiste donc à établir deux isotopies inhérentes distinctes selon le schéma suivant (qui correspond au schéma représentant la lecture passive):

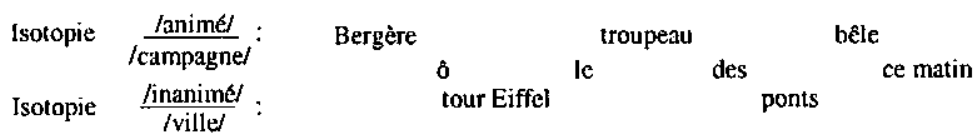

Ici, le parcours interprétatif comporte, en ce qui concerne les sémèmes, deux types d'opérations: (i) la réécriture de sémèmes de l'isotopie lexicalisée sur l'isotopie non lexicalisée; ex.: 'bêle' $\rightarrow$ |'klaxonne'|, où le sémème réécrit lexicalise le sème isotopant $(\mathrm{Cs})$ de l'isotopie non lexicalisée et des éléments du sémantème $(\mathrm{Sm})$ communs aux deux sémèmes (bêle $\rightarrow \mid$ 'klaxonne' $\mid \div \mathrm{Sm} / \mathrm{son} / \wedge \mathrm{Sm} /$ âpre/ $\wedge$ $\mathrm{Cs} /$ inanimé/); et (ii) l'association de sémèmes lexicalisés chacun sur une isotopie différente, en vue d'extraire leurs sèmes communs; ex.: 'Bergère' $\leftrightarrow$ 'tour Eiffel' (/verticalité/ ou /singularité/ sont de ces sèmes). Dans le graphique ci-dessous nous avons attribué à tous les sémèmes analysés les chiffres correspondant aux cas des sémèmes présentés sur le graphique illustrant la lecture active:

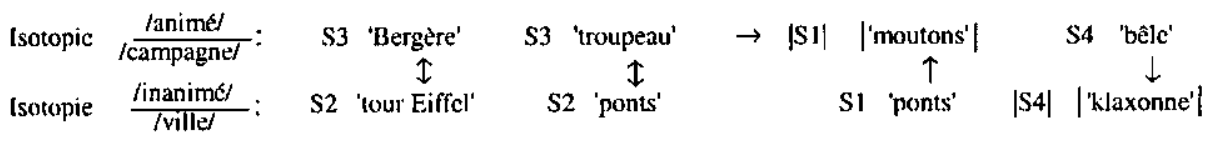

Les réécritures des sémèmes non lexicalisés et les extractions des traits communs aux sémèmes associés formulent des éléments d'une interprétation. Et la dernière étape de cette lecture active consistera dans la description des réseaux isotopiques établis par les isotopies horizontales et verticales, l'isotopie hyperonymique étant /ville/ $\wedge /$ campagne/ $\rightarrow$ /milieu/ ou /mode de vie/. 


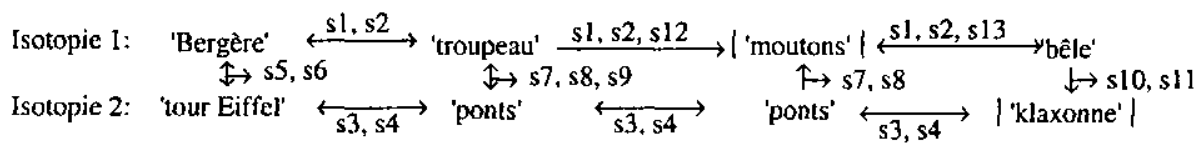
où: s1/animé/, s2/campagnc, s3/non animé/, s4/ville/, s5/verticalité/, s6/singularité/, s7/horizontalité/, s8/pluralité/, s9/ondulation/, s10/son/, sl1/âpre/, sl2/animaux domestiques/, s13/ovidés/

On remarquera que le $\mathrm{S}^{\mathrm{O}}$ 'ponts' participe à la fois à une connexion par réécriture et par association. On remarquera également une réécriture bilatérale en condensation 'troupeau des ponts' $\rightarrow$ 'moutons' $\leftarrow$ 'bêle' qui s'obtient par la lexicalisation de la molécule sémique composé de sèmes: $\mathrm{s} 1$, s12 (venant de 'moutons') et s 13 (venant de 'bêler').

Pour décrire une autre propriété importante de la lecture isotopique, nous rappellerons que les significations des sémèmes-occurrences peuvent être actualisées par assimilation du trait isotopant (cf. $n^{\circ} 2$ sème isotopant /humain/ assimilé par $S^{O^{\prime}}$ aboie' dans le contexte de $S^{O^{\prime}}$ commissaire'). Cette propriété nous renvoie au principe de présomption d'isotopie.

En général, on considère l'isotopie comme une forme remarquable de combinatoire sémique, un effet de la combinaison des sèmes. Ici au contraire, où l'on procède paradoxalement à partir du texte pour aller vers ses éléments, lisotopie apparâit comme un principe régulateur fondamental. Ce n'est pas seulement la récurrence de sèmes déjà donnés qui constitue l'isotopie, mais à l'inverse la présomption d'isotopic qui permet d'actualiser des sèmes, voire les sèmes. Cela apparâ̂t dans le processus d'identification des sémèmes: on distingue deux sémèmes différents dans casser un verre, $\mathrm{ct}$ dans boire un verre $e_{2}$ parce que le trait /rigidité/ dans 'casser' sélectionne le trait/rigidité/ dans 'verre', el le trait /liquidité/ dans 'boire' sélectionne /liquiditél dans 'verre' ${ }_{2}$. (Rastier 1987, p. 82).

Souvent, ces isotopies présumées peuvent être manifestées dans des séquences isotopes du texte éloignées les unes des autres. C'est, p.ex., le cas d'une lecture active d'un fragment de Vendredi ou les limbes du pacifique de M. Tournier, où l'auteur décrit le «long séjour» de Robinson dans une «grotte minuscule» passé en "position recroquevillée». Or, dans d'autres fragments du livre, on voit s'établir une isotopie inhérente /maternité/ et/ou /sexualité/. La lecture active du fragment sur la grotte nous permet donc de projeter cette isotopie sur les sémèmes du fragment analysé et de proposer leurs réécritures suivantes:

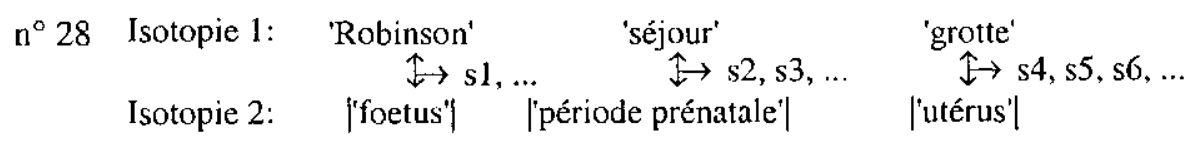

où: s1/position recroquevillée/, s2/temps/, s3/enfermé dans/, s4/cavité/, s5/sphéroïdité/, s6/contenant. 
Dans d'autres cas, l'isotopie présumée peut être extrinsèque au texte. C'est, p.ex., le cas de l'isotopie /sexualité/ établie dans le Petit chaperon rouge par une lecture psychanalytique.

La distinction entre isotopie intrinsèque et extrinsèque est établie relativement à une distinction analogue entre deux types d'interprétants et/ou d'interprétations. L'isotopie intrinsèque est constituée à la base d'une interprétation intrinsèque (et dans un interprétant intrinsèque) mettant en évidence les sèmes (inhérents ou afférents) actualisés dans un texte ou une séquence linguistique. L'isotopie extrinsèque est construite à la base d'une interprétation extrinsèque (et dans un interprétant extrinsèque) produisant des sèmes non actualisés dans le texte ou une séquence linguistique.

Le concept d'isotopie intrinsèque $v s$ extrinsèque ne fait pas l'objet principal de notre article, mais il convient de formuler trois remarques qui semblent importantes. En premier lieu, même si une isotopie extrinsèque peut paraître arbitraire, su projection sur le texte produira les mêmes opérations interprétatives et établira les mêmes relations sémantiques qu'en cas d'isotopies intrinsèques. En formulant des critères d'évaluation on remarquera cependant que plus une isotopie indexe de sémèmes produits par une interprétation extrinsèque, moins elle est valide. Ou plus exactement: l'évaluation de sa validité n'est plus du ressort de la sémantique (p.ex., l'isotopie /sexualité/ dans la lecture psychanalytique du Petit chaperon rouge). «La lecture qui "révèle « une isotopie entièrement ou principalement extrinsèque n'en perd pas pour autant son intérêt propre, ni, pourquoi pas, sa beauté»" ${ }^{6}$ (Rastier 1987, p. 242).

En second lieu, il s'avère donc que la validité ou la plausibilité de l'interprétation d'un texte dépend strictement de la connaissance de son interprétant intrinsèque (qui, outre le contexte linguistique du texte même, peut concerner également toutes sortes d'informations extratextuelles et extralinguistiques ou «encyclopédiques» nécessaires à une identification appropriée des significations

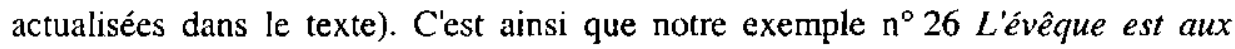
fideles comme le berger au troupeau peut être interprété comme «comparaison périlleuse (...) car si l'on a un mauvais esprit, [on peut] se représenter l'assemblée des fidèles comme un troupeau bêlant» (C. Kerbrat 1981, p. 177). Or, le «mauvais esprit» n'est, à nos yeux, rien d'autre qu'un interprétant extrinsèque dans lequel on

${ }^{6}$ Cf. cette interprétation de Deux amis de Maupassant ćtablie par Greimas suivant l'isotopie /religion/, où dans la séquence finale du récit les deux amis sont fusillés par des soldats prussiens et l'un, «plus grand», tombe "en travers" sur l'autre, ce qui pcrmet de réćcrire cette "position prospective des deux corps» par le sémème |croix'], et d'opérer d'autres réécritures intéressantes: au niveau des sémèmes: 'Morissot' $\rightarrow$ |'Jésus'|, |'ce martyre humain|", au niveau des séquences textuelles: «défaillance des deux amis à l'approche de la mort» $\rightarrow$ |«détresse de Jésus sur la croix»|, etc. (Greimas 1976, p. 238). 
procède à la virtualisation des traits actualisés dans son interprétant intrinsèque (normes de la culture chrétienne), et à l'actualisation d'autres traits tels que /grossièreté/ dans le premier couple, et /bêlant/ ou /stupidité/ dans le second, dans des interprétants caricaturaux ou satiriques. En somme, une identification correcte de tous les composants de l'interprétant intrinsèque, aussi bien linguistiques qu'extralinguistiques est une condition nécessaire de l'interprétation plausible des significations actualisées.

En troisième lieu, on remarquera enfin que le degré de complexité du parcours interprétatif permettant de (re)construire une isotopie est fonction notamment de la proportion relative des occurrences où le sème isotopant est inhérent, ou afférent: plus souvent le sème isotopant est inhérent, plus ce parcours est simple. On admettra aussi que plus grand est le nombre des traits non normatifs ou idiolectaux actualisés dans les sémèmes-occurrences dépendant d'une connaissance exacte de leur interprétant intrinsèque, plus la méconnaissance de cet interprétant peut provoquer l'incompréhension des significations actualisées. C'est pour cela que l'apparition des significations occasionnelles soumises à la spécificité de leur interprétant intrinsèque augmente la probabilité de leur assigner une signification non conforme aux intentions du locuteur. Et les significations des fragments de commentaires sportifs ci-dessous rendent parfaitement compte de l'écart pouvant exister entre les significations des mots actualisés dans leur interprétant intrinsèque et leurs réinterprétations extrinsèques.

$n^{\circ} 29$ Zidane fait un petit pont $(\mathrm{S} 1)$ au défenseur brésilien

$n^{\circ} 30$ Bartez a sorti une pêche(S2) infernale de l'attaquant brésilien de sa lucarne(S3)

Dans l'interprétant intrinsèque de \{commentaire d'un match de foot\} pour les significations actualisées dans les lexèmes mis en relief nous admettons respectivement Sl'passe de la balle entre les jambes', S2'balle qui va très rapidement', $S 3$ 'angle de la cage de but où se croisent le poteau et la transversale', tandis que dans un interprétant extrinsèque p.ex. dans une lecture humoristique, nous lirons ces significations comme: Sl'petite construction reliant deux points audessus d'une dépression', S2'fruit du pêcher', S3'petite fenêtre'. Les réinterprétations suggérées sont cependant extrinsèques et s'appuient sur la réactualisation des traits virtualisés dans l'interprétant intrinsèque, et leur effet comique résulte du fait que nous connaissons les significations réellement actualisées. Si donc la deuxième interprétation est également possible, elle ne sera valide ou plausible que dans l'interprétant extrinsèque. Dans l'interprétant intrinsèque seule la première est plausible. Ce qui mène à rappeler cette évidence que même si toute lecture est possible, toute interprétation n'est pas plausible. 


\section{CONCLUSION}

Dès sa première apparition dans un chapitre de la Sémantique structurale de Greimas (1966) le concept d'isotopie a fait l'objet de maintes recherches et s'est répandu à tous les domaines des «sciences de signification». Une simple idée de l'itérativité d'un même thème dans différentes unités de signification a donné lieu à différentes classifications d'isotopies, de textes poly-isotopes et de stratégies interprétatives. On distingue des isotopies discursives et systémiques, des isotopies sémantiques, grammaticales, phonétiques. Le concept d'isotopie s'étend à d'autres dimensions sémiotiques: on l'utilise pour une analyse des dessins (Budor 1978), pour une analyse des images et des films (Odin 1990). Il sert à décrire les mécanismes d'assignation des significations aux mots dans l'interprétant intratextuel - isotopie intrinsèque - et dans l'interprétant extratextuel - isotopie extrinsèque. A rebours de la classification des isotopies, il permet également une classification des textes. Un texte qui ne comporte qu'une isotopie générique est un texte univoque, celui qui en comporte plusieurs, un texte plurivoque et plus grand sera le nombre d'isotopies génériques homopositionnelles et le nombre des sémèmes indexés sur elles, plus le texte sera plurivoque. On imagine alors que les textes scientifiques représenteraient des textes mono-isotopes, dont les mots ne se rapporteraient qu'à un domaine sémantique bien déterminé et qui tendraient à éliminer des afférences, tandis que la poly-isotopie serait une propriété des textes poétiques permettant d'affecter, par réécriture ou par association, une seule et même unité lexicale à différentes isotopies génériques.

Mais la plus grande utilité du concept d'isotopie semble résider dans son approche opérationnelle. De ce point de vue, il permet de rendre compte de manière scientifique et objective des lectures assignées «intuitivement» (sans avoir conscience des opérations effectuées) au texte qui s'inscrit dans tout acte de parole le plus ordinaire (p.ex. blagues, énoncés publicitaires), ainsi que de proposer des outils pour une interprétation plausible de textes beaucoup plus complexes, dont la signification ne nous est pas donnée de manière aussi évidente (p.ex. textes poétiques). Ainsi, le concept d'isotopie permet de donner une description cohérente non seulement des relations sémantiques établies dans un texte interprété sous son angle, mais en plus une description du processus d'interprétation rendant compte de règles d'assignation de la signification à un texte, et permettant de même de ménager la possibilité - souvent contestée par des sémanticiens - d'une description scientifique de l'interprétation, et de son objectivation.

\section{BIBLIOGRAPHIE}

Bcaugrande, R.-A. de, Dressler, W.-U. (1990), Wstęp do lingwistyki tekstu, Warszawa.

Budor, K. (1978), Dibujo humoristico y chiste verbal: ensayo de analisis semantico, Studia Romanica et Anglica Zagrabiensia, 23. 
Corblin, F. (1996), Sémantiques du discours, Langages 123.

Florczak, J. (2002), O wybranych elementach znaczenia tekstu $w$ mediach $w$ perspektywie semantyki interpretacyjnej. Matériaux du colloque, w: Tekst w mediach, Lódź, Wyd. UŁ, ss. 3645.

Grcimas, A.-J. (1966), Sémantique structurale, Paris.

Greimas, A.-J. (1976), Maupassant. La sémiotique du texte, Paris.

Groupe $\mathrm{Mu}$ (1976), Isotopie et allotopie. Le fonctionnement rhétorique du texte, Versus, 14.

Groupe Mu (1974), Lecture du poème et isotopies multiples, Le français moderne, 42.

Kerbrat-Orccchioni, C. (1976), Problématique de l'isotopie, Linguistique et sémiologie.

Kerbrat-Orecchioni, C. (1981), Des usages comigues de l'analogie, Folia Linguistica, XV.

Klinkenberg, J.-M. (1973), Le concept d'isotopie en sémantique et en sémiotique littéraire, Le français moderne, 41.

Odin, R. (1990), Cinéma et production de sens, Paris.

Rastier, F. (1987), Sémantique interprétative, Paris.

Rastier, F. (1985), L'isotopie sémantique, du mot au texte, Paris.

Sperber, D., Wilson, D. (1986), Relevance: Communication and Cognition, Oxford.

Weinreich, U. (1966), Explorations in Semantic Theory, in: Current Trends in Linguistics, Den Haag. 